\title{
Metal sensitivity in patients with a hinge arthroplasty of the knee
}

\author{
M. WEBLEY, A. KATES, AND M. L. SNAITH \\ From the Department of Rheumatology, St Stephen's Hospital, London
}

SUMMARY Fifty patients who had received a hinge arthroplasty of the knee were investigated for possible metal sensitivity. Patients were patch tested against all the metal constituents of the prosthesis. Positive patch tests were found in $32 \%$ of patients. Seventeen patients had either loosening or a persistent sterile discharge from the knee. No correlation was found between these complications and metal sensitivity. It was concluded that metal sensitivity is probably not a primary factor in the pathogenesis of complications, particularly loosening.

Patients who have metal on metal prostheses have been shown to have a raised incidence of metal sensitivity as demonstrated by patch tests (Benson et al., 1975; Elves et al., 1975). This has been suggested as a possible cause of loosening in these prostheses (Evans et al., 1974; Benson et al., 1975; Jones et al., 1975). Prosthetic joints shed a considerable number of metallic particles (Charoskey et al., 1973) and patients have elevated blood levels and a raised urinary excretion of cobalt (Coleman et al., 1973). If metal sensitivity were a primary event in the production of loosening or other complications such as sterile discharge, positive skin tests might be expected in these cases. Previous studies (Evans et al., 1974; Benson et al., 1975; Elves et al., 1975; Jones et al., 1975; Brown et al., 1977) have been mainly in hip replacements. We decided to investigate our patients who had received a hinge arthroplasty of the knee to determine the incidence of metal sensitivity and to assess whether any correlation existed with complications which had occurred.

\section{Patients and methods}

Fifty patients with a hinge arthroplasty of the knee either of the Walldius or Guepar type were studied. These prostheses were inserted between 1971 and 1975 and all patients who were able to attend in 1976 were investigated.

Thirty-nine patients had rheumatoid arthritis and 11 had osteoarthrosis.

Accepted for publication November 7, 1977

Correspondence to Dr M. Webley, Department of Rheumatology, Westminster Hospital, Horseferry Road, London SW1P 2A.P
Thirty-three control patients, with either rheumatoid arthritis or osteoarthrosis, but without prostheses were also tested.

The ages of the patients ranged from 44 to 76 years (mean 66) and the ages of the control group ranged from 47-76 years (mean 64).

Thirty-eight of the patients were women and 12 were men. In the control group, 26 were women and 7 were men.

A history of allergy was sought with particular reference to metals. An occupational history was also taken. Patients were patch tested using ' $\mathrm{AL}$ ' test strips to which the allergens were applied. The test strips were placed on the the patients' backs and held in place with micropore tape.

All the metal constituents of the prostheses were tested. These were cobalt chloride $2 \%$, nickel sulphate $5 \%$, and potassium dichromate $0.5 \%$ (obtained from the Trolle Lassen Laboratories, Hellerup, Denmark). Manganese $2 \%$, silicon $2 \%$, iron $2 \%$, and molybdenum $1 \%$, made up in white paraffin. In addition, patients were tested against the acrylic polymer $1 \%$ and $10 \%$ and cement $1 \%$ and $10 \%$.

The patch test sites were read at 72 hours. Responses were graded as follows grade I-erythema only; grade II-erythema and vesicles; grade IIIerythema and vesicles extending beyond the patch sites. To exclude false positive results from local irritation, patients were also seen at 1 week and the test sites re-examined.

\section{Results}

Table 1 shows the number of positive responses to 
cobalt, chromium and nickel. These occurred in a total of 17 patients. Eight patients had a reaction to cobalt only. One patient, who was known to have nickel sensitivity, reacted to this metal alone-for this reason she was excluded from further analysis. The other 8 patients all had 2 or more positive responses to the metal allergens. Cobalt was positive in 7 of these, chromium in 4, and nickel in 4. Three patients had a positive response to manganese and 1 patient had a reaction to cement. These reactions were only present at 72 hours and were considered to be non-specific. Three patients $(9 \%)$ in the control group had a positive patch test, all to cobalt.

Table 2 shows the correlation between positive patch tests and complications. Seven had loosening of the prosthesis alone and 10 had a persistent sterile discharge from the knee with a minor degree of loosening of the prosthesis. Sixteen of the 50 patients $(32 \%)$ had a positive patch test to one or more of the allergens. Of the 7 patients with loosening, only $1(14.3 \%)$ had a positive result. Of the 10 patients with a persistent discharge, $5(50 \%)$ had a positive reaction. There were 6 patients with loosening and 5 patients with a persistent discharge who had a negative reaction. There were 33 patients with no complications. Ten of these $(30 \%)$ had a positive patch test. Eight patients had a grade I reaction to cobalt. Four patients had a grade II reaction and 3 patients had a grade III response. None of the patients with grade II reactions had complications, whereas all those with a grade III reaction did.

Table 1 Allergens used for patch testing

\begin{tabular}{lrr}
\hline Allergens & & No. of positive results \\
\hline Nickel sulphate & $5 \%$ & 5 \\
Cobalt chloride & $2 \%$ & 15 \\
Potassium dichromate & $0.5 \%$ & 4 \\
Manganese & $2 \%$ & 3 \\
Silicon & $2 \%$ & - \\
Iron & $2 \%$ & - \\
Molybdenum & $1 \%$ & - \\
Acrylic polymer & $1 \%$ & - \\
Acrylic & $10 \%$ & - \\
Cement & $1 \%$ & 1 \\
Cement & $10 \%$ & - \\
\hline
\end{tabular}

Table 2 Results of patch testing

\begin{tabular}{lllll}
\hline $\begin{array}{l}\text { Patients } \\
(n=50)\end{array}$ & $\begin{array}{l}\text { No compli- } \\
\text { cations } \\
(n=33)\end{array}$ & $\begin{array}{l}\text { Loosening } \\
(n=7)\end{array}$ & $\begin{array}{l}\text { Discharge } \\
(n=10)\end{array}$ & $\begin{array}{l}\text { All compli- } \\
\text { cations } \\
(n=17)\end{array}$ \\
\hline $\begin{array}{c}\text { Positive test } \\
(n=16)\end{array}$ & $10(30 \%)$ & $1(14 \cdot 3 \%)$ & $5(50 \%)$ & $6(35 \cdot 5 \%)$ \\
$\begin{array}{c}\text { Negative test } \\
(n=34)\end{array}$ & 23 & 6 & 5 & 11 \\
\hline
\end{tabular}

Table 3 Time after surgery of patch testing

\begin{tabular}{lrll}
\hline Years & No. of patients & Positive patch test & \\
\hline 1 & 9 & 3 & 5 \\
2 & 16 & 6 & $\overline{5}$ \\
3 & 9 & 2 & \\
4 & 11 & 4 & \\
5 & 5 & 1 & 0 \\
\hline
\end{tabular}

Table 3 shows the time after insertion of th $\frac{\mathbb{\Phi}}{8}$ prostheses that patch testing was carried out. It caf be seen that positive reactions were not related to the length of time that the prosthesis had been in place. Positive reactions occurred in 3 patients studiet within 1 year of surgery. Patients with rheumatoi arthritis and osteoarthrosis had a similar incidencỗ of positive patch tests.

\section{Discussion}

The incidence of cobalt sensitivity of $9 \%$ in oup control group of patients is similar to that in the general population in Europe (Cronin and Wilkin son, 1973). Our results in patients with hinge arthroplasties of the knee show a significantlep increased overall incidence of metal sensitivity $(32 \%)$. This is in keeping with the findings of EQes: et al. (1975) who found an incidence of $38 \%$ in patients with total joint replacements. Benson e (1975) found an incidence of metal sensitivity? $28 \%$ in patients with McKee hip replacements bu only $2.6 \%$ in patients with Charnley hips, suggesting that metal sensitivity is far more likely to occur in patients with metal on metal prostheses. Like them $\vec{\delta}$ we found that cobalt was the principal metal agains\$ which patients reacted; nickel and chromiun accounting for only a small number of positive reactions.

Benson et al. (1975) also suggested an associatio between metal sensitivity and loosening of metal oo metal prostheses. Evans et al. (1974) found a strong. correlation between patch tests and loosening. AQ the metal sensitive patients in their series had prostheses which were loose. Similarly, Jones et at (1975) found that 6 out of 7 patients with complicate McKee hip arthroplasties had a positive reaction te. cobalt. Brown et al. (1977), however, found no positive patch tests in 20 patients with loose McKeeFarrar hips, despite using two different preparations of cobalt. In our patients, despite the high overad incidence of metal sensitivity, we were unable to show any correlation with loosening. A positike patch test occurred in only 1 out of 7 patients witw a loosened prosthesis.

Previous studies (Evans et al., 1974; Benson et a 1975; Elves et al., 1975; Jones et al., 1975; Brow et al., 1977) have been mainly in patients with hig 
replacements. Evans et al. (1974) included only 1 patient who had a bilateral knee arthroplasty in their series and Elves et al. (1975) included only a small number of knee replacements in their series of complicated arthroplasties. It might be that there are important differences between total hip and total knee replacements, particularly with regard to surface area and wear. This might explain variations in the incidence of positive patch tests in different series.

Metal on metal prostheses shed a considerable number of metallic particles (Charoskey et al., 1973) and it has been shown that these are taken up by macrophages (Heath et al., 1971). Rae (1975) has demonstrated in vitro that macrophages exposed to cobalt become damaged, undergo toxic changes, and allow the release of lactic acid dehydrogenase. This process would provide an explanation for the necrotic tissue surrounding prosthetic joints and it seems possible that this is the cause of a persistent sterile discharge from the joint. This complication occurred in 10 of our patients and it is interesting that 5 of these had a positive patch test against cobalt.

Uptake of metal particles by macrophages might be expected to sensitise patients with the production of a positive patch test. If this was the case, cellmediated immunity to cobalt and other metals might be demonstrable in vitro. Lymphocyte transformation has been shown in vitro with nickel (Macleod et al., 1970) and chromium (Tio, 1976) but further studies are clearly necessary using cobalt to clarify the situation still further. It seems from our findings that non-specific local tissue reaction is just as likely as metal sensitivity, to be the primary reason for loosening and sterile discharge. Metal sensitivity as reflected by a positive patch test may be only a secondary phenomenon and this would explain the observations of positive reactions in patients with uncomplicated arthroplasties.

We would like to thank Dr Jonathan Brostoff for his helpful advice and useful comments. We would also like to thank Dr E. Cronin for her helpful advice on patch testing techniques.

\section{References}

Benson, M. K. O., Goodwin, P. G., and Brostoff, J. (1975). Metal sensitivity in patients with joint replacement arthroplasties. British Medical Journal, 4, 374-375.

Brown, G. C., Lockshin, M. D., Salvak, E. A., and Bullough, P. G. (1977). Sensitivity to metal as a possible cause of sterile loosening after cobalt-chromium total hip replacement arthroplasty. Journal of Bone and Joint Surgery, 59A, 2, 164-168.

Charoskey, C. B., Bullough, P. G., and Wilson, P. D. (1973). Total hip replacement failures, a histological evaluation. Journal of Bone and Joint Surgery, 55A, 49-58.

Coleman, R. F., Herrington, J., and Scales, J. T. (1973). Concentration of wear products in hair, blood and urine after total hip replacement. British Medical Journal, 1, 527-529.

Cronin, E., and Wilkinson, D. (1973). Recent Advances in Dermatologv, No. 3, pp. 134-192. Ed. by A. Rook. Churchill Livingston, London and Edinburgh.

Elves, M. W., Wilson, J. N., Scales, J. T., and Kemp, H. B. S. (1975). Incidence of metal sensitivity in patients with total joint replacements. British Medical Journal, 4, 376-378.

Evans, E. M., Freeman, M. A. R., Miller, A. J., and Vernon Roberts, B. (1974). Metal sensitivity as a cause of bone necrosis and loosening of the prosthesis in total joint replacement. Journal of Bone and Joint Surgery, 56B, 626-642.

Heath, J. C., Freeman, M. A. R., and Swanson, S. A. V. (1971). Carcinogenic properties of wear particles from prostheses made in cobalt-chromium alloy. Lancet, 1, 564-566.

Jones, A. D., Lucas, H. K., O'Driscoll, M., Price, C. H. G., and Wibberley, B. (1975). Cobalt toxicity after McKee hip arthroplasty. Journal of Bone and Joint Surgery, 57B, 3, 289-296.

Macleod, T. D., Hutchinson, F., and Raffle, E. J. (1970). The uptake of labelled thymidine by leucocytes of nickel sensitive patients. British Journal of Dermatology, 82, 487-492.

Rae, T. (1975). A study on the effects of particulate metals of orthopaedic interest on murine macrophages in vitro. Journal of Bone and Joint Surgery, 57B, 4, 444-450.

Tio, D. (1976). A study on the clinical application of a direct leucocyte migration test in contact allergy. British Journal of Dermatology, 94, 65-70. 